\title{
Validation of the 8-Item Morisky Medication Adherence Scale in Chronically Ill Ambulatory Patients in Rural Greece
}

\author{
Sotirios Plakas1,2, Dimos Mastrogiannis ${ }^{3}$, Marianna Mantzorou1, \\ Theodoula Adamakidou, ${ }^{1,2}$, Georgia Fouka1,2, Angeliki Bouziou ${ }^{4}$, Chrysoula Tsiou, \\ Donald E. Morisky 5 \\ ${ }^{1}$ Department of Nursing, Technological Educational Institution (TEI) of Athens, Athens, Greece \\ ${ }^{2}$ Research Unit for Nursing Rehabilitation of the Chronically III, Technological Educational Institution (TEI) of \\ Athens, Athens, Greece \\ ${ }^{3}$ Department of Nursing, Technological Educational Institute of Central Greece, Lamia, Greece \\ ${ }^{4}$ General Hospital of Aitoloakarnania, Agrinion, Greece \\ ${ }^{5}$ Department of Community Health Sciences, UCLA Fielding School of Public Health, Los Angeles, CA, USA \\ Email: skplakas@teiath.gr,dmastrogiannis@teilam.gr,mantzorou@teiath.gr, adamakidou@yahoo.gr, \\ foukageo@hotmail.com, moutsino@hotmail.com,chrisa-tsiou@hotmail.com,dmorisky@ucla.edu
}

Received 6 February 2016; accepted 8 March 2016; published 11 March 2016

Copyright (C) 2016 by authors and Scientific Research Publishing Inc.

This work is licensed under the Creative Commons Attribution International License (CC BY).

http://creativecommons.org/licenses/by/4.0/

c) (i) Open Access

\begin{abstract}
Objective: To validate a Greek version of the structured self-reported 8-item Morisky Medication Adherence Scale (MMAS-8) and determine its psychometric properties in patients with chronic illnesses. Methods: A cross-sectional survey was conducted in a small public hospital and a public health care centre, in a rural town in western Greece. The sample consisted of 100 patients with various chronic illnesses. Data were collected between January-May 2011, on the Greek version of the MMAS-8 and Beliefs about Medicines Questionnaire (BMQ). Results: Scale's reliability analysis revealed an overall Cronbach's alpha of 0.753 and the corrected item to total correlations, were greater than 0.30 for each of the 8 items comprising the medication adherence scale, showing good internal consistency. Convergent validity was supported by a significant correlation between the present scale's total score and the BMQ-Specific Necessity score (Spearman's rho $=0.492, p<$ 0.001). Conclusion: The current study showed acceptable reliability and validity of the Greek version of the 8-item MMAS to measure adherence to medications for various chronic illnesses. The validated Greek version of the MMAS-8 can help towards understanding adherence barriers in Greece so as to develop effective strategies to increase adherence and reduce the costs.
\end{abstract}

\section{Keywords}

Adherence, Validation, Chronic Illnesses, Morisky MMAS-8 Scale 


\section{Introduction}

One of the most important issues in the medical treatment of chronic illnesses is patient adherence to medications. Adherence is the degree to which a person's behaviour coincides with medical advice [1]. Adherence to prescribed medicines is a way to improve one's health, assuming the prescription is appropriate and correct.

A growing body of literature on patient adherence has shown that a large proportion of patients are non-adherent ranging from as low as $6.3 \%$ to as high as $87 \%$ [2]-[6].

In a recent report of the ABC (Ascertaining Barriers for Compliance) project in Europe, in a sample of 2630 hypertensive patients from 11 EU countries, which used the 4-item Morisky scale for primary outcome measure and the MARS scale for secondary outcome measure, $42 \%$ were found to be non-adherent to their medication regime while for $18 \%$ of them that was intentional [7]. It was also found that adherence rates varied considerably among countries, as 34\% of hypertensive patients were non-adherent in Austria and 70\% were non-adherent in Hungary [8]. In another study in Northern Ireland using the Reported Adherence to Medications scale (RAM), only $9.3 \%$ of hypertensive patients were found to be non adherent [9].

In Greece, different studies with different methodologies to estimate adherence among hypertensive patients have shown conflicting results. A study using the 4-item MMAS has reported that $74 \%$ of hypertensive patients were adherent to their medications [10]. In another study [11] most of the participants (77.1\%) reported they received their medication according to their physician's directions while statistically significant correlations were found between the mean systolic blood pressure values and compliance with drug treatment $\left(\mathrm{r}_{\mathrm{pb}}=-0.331, p<\right.$ $0.01)$.

On the other hand, other researchers [12] measuring adherence based on a number of questions about regular use of medications, reported that only $15 \%$ of hypertensive patients were adherent.

This discrepancy in research evidence regarding medication adherence in Greece, needs further exploration because poor adherence has not only been related with poorer patient outcomes, illness complications, and higher morbidity [5] [12] but poor adherence can also cause direct and indirect increases in costs to the health care systems [14].

Greece is currently experiencing severe fiscal problems and severe austerity measures are in effect, with the aim to reduce the public dept by reducing health expenditure among other measures [15]. In 2008, per capita spending for pharmaceutical products in Greece was the highest among European countries in 2008 amounting to 700 US dollars per person [15]. Thus, reducing expenses for pharmaceutical products is a major target for the Greek government. According to Karamanoli [16], medical expenses were 5.2 billion euros in 2009 and were dropped to 1.65 billion euros in 2011 following the measures taken. However, as Vandoros and Stargardt [15] point out, those measures should not undermine quality of care for the Greek patients; instead there is a need to increase efficiency in medicines' use. A valid estimation of the problem of adherence in Greece is imperative for the development of effective strategies to improve adherence to medications as adherence is one way to improve efficiency of the medicines used. Also, understanding adherence barriers is necessary to determine strategies to improve medication adherence and achieve optimum outcomes.

Several factors have been linked with low compliance to prescribed medicines, with the main ones being the patient himself, the condition of the illness, the management of the disease, the socioeconomic status of the patient and health care system/health professionals factors [6]. In addition, according to the European report mentioned above, low perceived self-efficacy, high perceived barriers, cost related behaviour, adverse drug reactions and frequency of dosing, were the main factors associated with poor non-adherence [7].

Even though a variety of methods are available to estimate adherence, patient related factors of adherence are the most frequently reported in the literature [6]. High tech instruments have been developed that can electronically record every opening of a tablet bottle but there are concerns about both the cost of them and the autonomy of patients regarding their decisions about their own health care [17]. Besides, as non-adherence is both intentional and unintentional, a single instrument or device cannot be effective when two different kinds of behaviour can affect adherence. Furthermore, those instruments may not be suitable in every patient situation [18] while there is no evidence on the cost effectiveness of such devices [19].

Pharmacy fill data and biomarkers are other objective ways to estimate adherence, but more practical and easily available measurements of adherence are needed in the busy clinical settings. This is even more necessary for Greece where financial resources are very limited and an inexpensive measurement of adherence is mostly needed. 
The most frequently used method to measure adherence is by self reported scales [18] [20]. Self reported instruments are convenient, inexpensive, easy to administer and effective [18].

The most frequently used self-reported questionnaire, is the Morisky Medication Adherence Scale (MMAS) and recently a new 8-item MMAS has been established in patients with hypertension [21]-[23]. The 8-item MMAS has been validated for use in several countries with several groups of patients and has been found to be a valid and reliable scale to measure adherence in hypertensive, diabetic, osteoporosis, epilepsy, myocardial infarction and patients taking warfarin [14] [18] [20] [24]-[32].

The wide usage of the scale in the international literature triggered our interest to translate and validate the 8-item MMAS in Greece, in patients with various chronic disorders. Thus the aim of this study was to validate a Greek translation of the structured self-report 8-item Morisky Medication Adherence Scale (MMAS-8) and determine its psychometric properties in patients with chronic illnesses. In addition, to measure adherence and explore sociodemographic factors that influence adherence.

\section{Methods}

\subsection{Sample and Design}

A cross sectional survey was conducted in a public General Hospital with approximately 13,000 patient admissions per year and a public health care centre in a rural town in western Greece. The town has nearly 95,000 inhabitants while the hospital has 150 beds. Sampling was based on convenience, involving any chronically ill patient who was attending a nephrology and internal medicine outpatient clinics of the hospital and the health care centre to obtain their monthly reimbursable medical prescriptions. It is important to mention that in this setting we were unable to differentiate the patients according to their health condition. In Greece, patients may visit a medical practitioner privately for a special condition but usually those doctors are not contracted with every social insurance fund and cannot issue reimbursable medical prescriptions. As a result, patients must attend the public hospital or health centres where doctors are allowed to issue reimbursable medical prescriptions for most of the medicines for almost every social insurance fund. For this reason, our sample consisted of patients with multiple chronic conditions.

Furthermore, as the patients usually visit the clinic just to renew their prescription, doctors do not keep medical records for all patients so, all information about the health condition and medications for those patients, were collected from the patients themselves.

To avoid the social desirability bias, all data were collected by a junior doctor doing a clinical rotation in primary care medicine, while the doctors of those primary care clinics had no access to the data. The patients preferred the questions to be read to them by the researcher, therefore all data were collected on a person to person structured interview.

\subsection{Data Collection}

Data were collected between January-May 2011, on the Greek version of MMAS-8, on Beliefs about Medicines Questionnaire (BMQ) and a questionnaire with various sociodemographic and illness related data.

Every adult patient attending the clinics was approached, was explained the purpose of the research and was asked to participate after the end of the visit to the doctor. Inclusion criteria for the patients were to be able to speak Greek and have at least one chronic disease treated with medications for a year or more. Sociodemographic data included age, gender, educational level, marital status, income, social security coverage fund, kind of disease, duration of disease, number of medications etc.

An adequate sample size was estimated to be 80 patients for this reliability and validity study. The justification for the sample size was based on the recommendation by Hutcheson and Sofroniou [33] that size should be ten times the number of the items of the questionnaire. We aimed to double this size to increase the accuracy of the validation outcomes. However, approximately more than half of the patients approached, declined to participate, citing reasons such as lack of time and unwillingness. A total of 100 questionnaires were completed which was sufficient to detect a moderate difference in adherence with a type 1 error at 0.05 and a power of .80 , a number considered to be adequate for the purposes of this study.

\subsubsection{The 8-Item MMAS}

This self-report scale consists of 7 items answered with a yes or no and 1 item with a 5-point Likert scale. The 
scores of the MMAS-8 range from 0 to 8 . A score below 6 indicates low adherence, a score between $6<8$ medium adherence and a score of 8 high adherence. The 8-item MMAS has established a second level criterion related validity, following comparisons with pharmacy fill rates [22] [26].

In addition, the 8-MMAS scores have been found to be significantly related with physiological effects such as hypertension control and HbA1c levels [14] [24] [25] [27] However, using the HbA1c levels and hypertension control as a gold standard, the criterion related validity was found to be poor to moderate [14] [24] [27].

\subsubsection{Beliefs about Medicines Questionnaire (BMQ)}

The beliefs about medicines questionnaire (BMQ) was developed by Horne, Weinman and Hankins in 1999. The BMQ scale is consisted of two sections: The BMQ-Specific, which concerns prescribed medications and the BMQ-General which concerns beliefs about medicines in general. The BMQ-Specific is consisted of 5 items assessing beliefs about the necessity of prescribed medication (Specific-Necessity) and 5 items assessing concerns about prescribed medication (Specific-Concerns). The BMQ-General is consisted of two 4 items subscales which concerns beliefs that medicines are harmful, (General-Harm) and concerns that medicines are overused by doctors (General-Overuse) [34] [36].

Patient's beliefs about their medications is one factor that is strongly related with adherence [1] [34] [35]. Two areas of medication beliefs are considered to be the most important factors affecting adherence. This is the beliefs about the necessity of their medications and the concerns about the negative consequences of their medications [36]. Higher scores on each area indicate stronger beliefs about either the necessity domain of their medications or the concerns domain for their medications.

\subsubsection{Translation and Cross-Cultural Adaptation of MMAS-8}

A standard translation and back translation procedure was undertaken. Two qualified independent translators performed a forward translation of the original MMAS-8 into Greek, without any mutual consultation. A consensus meeting between the researchers (nurses and medical doctors) established the cross cultural adaptation of the instrument. A third translator performed a reverse translation from Greek into English. Then, the original and the back translated versions were evaluated in another consensus meeting between the researchers in which any variations were resolved and the final Greek version of the MMAS-8 was established.

The translated version of this paper was compared with the one produced by the MAPI institute; it was found equivalent and was approved by the owner of the scale.

The final Greek version of the MMAS-8 was tested on 10 patients and no problems were identified in understanding the questions. Those 10 questionnaires were not included in this study.

\subsection{Ethics}

An ethical approval was given by the appropriate scientific committee of the clinical settings. The study procedures were described to participants, consent was obtained and they were interviewed on location. All patients were assured that their responses would be confidential and that they could withdraw from the study at any time they wished, without any consequences.

\subsection{Statistical Analyses}

We used exploratory factor analysis with SPSS v.19.0 in order to examine the scale's reliability in the present population. Construct validity, which is the measure of how well a scale is actually measuring the construct or concept it is supposed to measure, is provided by evidence of convergent and divergent validity. The former, since the present scale did not identify any subscales, can be calculated by multi-item analysis which reveals the same results with the corrected item to total correlations, depicted in Table 3. Except for item "Did you take your medications yesterday?" that yielded a value of 0.391, all remaining items are above the 0.4 threshold which is considered adequate for this analysis [37]. Convergent validity was examined with Spearman correlation calculations between the scores of the MMAS-8 and the scores of the domains of necessity and concerns of the BMQ-Specific. Divergent validity could not be established, since, participants weren't asked to fill in any other scale measuring a different construct/concept.

Mann-Whitney and Kruskal-Wallis tests were conducted to evaluate the possible associations between various sociodemographic factors with medication adherence which are presented in the results of the study. 


\section{Results}

\subsection{Sample Characteristics}

Table 1 depicts the sociodemographic characteristics of the participants in the present study. The mean age of participants was 63.79 years $(\mathrm{SD}=14.89$ years $) .51 \%(\mathrm{n}=51)$ were female, $65.7 \%(\mathrm{n}=65)$ were married, $45.7 \%$ $(n=43)$ had three children, and 53.1\% $(n=52)$ had finished primary school, $67.3 \%(n=66)$ of the sample reported under $€ 16,000$ yearly income.

Table 1. Sociodemographic characteristics of the participants.

\begin{tabular}{|c|c|c|}
\hline & Mean (SD) & Number (percentage) \\
\hline Age & 63.79 (14.89) & $\mathbf{n}=\mathbf{1 0 0}$ \\
\hline Gender & & $\mathrm{n}=\mathbf{1 0 0}$ \\
\hline Male & & $49(49)$ \\
\hline Female & & $51(51)$ \\
\hline Nationality & & $\mathbf{n}=\mathbf{9 7}$ \\
\hline Greek & & $96(99)$ \\
\hline Other & & $1(1)$ \\
\hline Marital status & & $n=99$ \\
\hline Married & & $65(65.7)$ \\
\hline Single & & $10(10.1)$ \\
\hline Divorced & & $4(4.0)$ \\
\hline Widower & & $20(20.2)$ \\
\hline Number of children & & $\mathrm{n}=94$ \\
\hline 0 & & $6(6.4)$ \\
\hline 1 & & $6(6.4)$ \\
\hline 2 & & $16(17.0)$ \\
\hline 3 & & $43(45.7)$ \\
\hline$>3$ & & $23(24.5)$ \\
\hline Income & & $\mathrm{n}=\mathbf{9 8}$ \\
\hline$<10.000$ & & 33 (33.7) \\
\hline $10.000-15.000$ & & 33 (33.7) \\
\hline $16.000-20.000$ & & $7(7.1)$ \\
\hline $21.000-25.000$ & & $16(16.3)$ \\
\hline $26.000-30.000$ & & $7(7.1)$ \\
\hline$>30.000$ & & $2(2.0)$ \\
\hline Level of education & & $\mathrm{n}=\mathbf{9 8}$ \\
\hline Primary school & & $52(53.1)$ \\
\hline High school & & $12(12.2)$ \\
\hline Lyceum & & $17(17.3)$ \\
\hline TEI/AEI & & $17(17.3)$ \\
\hline Social insurance coverage & & $\mathrm{n}=\mathbf{9 8}$ \\
\hline IKA & & 27 (27.6) \\
\hline OGA & & $55(56.1)$ \\
\hline Public & & $12(12.2)$ \\
\hline Other & & $4(4.1)$ \\
\hline
\end{tabular}


Patients had complex chronic illnesses, with $80 \%(\mathrm{n}=80)$ having more than two co-morbidities. More specifically, 20\% $(n=20)$ had only one chronic condition, 28\% $(n=28)$ had 2 chronic conditions, $37 \%(n=37) 3$ conditions, $13 \%(n=13) 4$ conditions and $2 \%(n=2) 5$ conditions.

Hypertension was the most common condition with $79 \%(n=79)$, followed by diabetes $36 \%(n=36)$, coronary heart disease $18 \%(n=18)$, dyslipidaemia $18 \%(n=18)$, and osteoporosis $15 \%(n=15)$, while $21 \%(n=21)$ had chronic renal failure requiring dialysis and $7 \%(\mathrm{n}=7)$ had renal failure not requiring dialysis.

More than $90 \%(\mathrm{n}=90)$ of the patients were on more than 3 different medications a day. The mean score for the medication adherence scale was 4.216 (standard deviation $=2.05$ ).

\subsection{Validity and Reliability}

Initial analysis of data showed that there wasn't any significant multicollinearity (determinant of correlation matrix $=0.067$ ). The Kaiser-Meyer-Olkin measure of sampling adequacy was 0.724 which, according to Hutcheson and Sofroniou [33] it is a good figure. Also, for these data, Bartlett's test of sphericity was highly significant ( $p$ $<0.0001$ ), indicating the appropriateness of factor analysis. Exploratory factor analysis revealed that the eight-item scale was unidimensional and the items loaded well on a single factor (Table 2). Scale's reliability analysis revealed an overall Cronbach's alpha of 0.753 . The corrected item to total correlations, were greater than 0.30 for each of the 8 items comprising the medication adherence scale, showing good internal consistency (Table 3).

Convergent validity was supported by a significant correlation between the MMAS-8 total score and the BMQ-Specific Necessity score (Spearman's rho $=0.492, p<0.001$ ).

Table 2. Factor loadings of the eight-item medication adherence scale.

\begin{tabular}{cc}
\hline Item & Factor loadings \\
\hline How often do you have difficulty remembering to take all your medications? & 0.867 \\
When you feel like your condition is under control, do you sometimes stop taking your medicines? & 0.819 \\
Have you ever cut back or stopped taking your medication without telling your doctor, \\
because you felt worse when you took it? \\
Do you sometimes forget to take your medications? & 0.742 \\
People sometimes miss taking their medications for reasons other than forgetting. & 0.733 \\
Thinking over the past two weeks, were there any days when you did not take your medicines? & 0.654 \\
When you travel or leave home, do you sometimes forget to bring along your medications? & 0.575 \\
Taking medication everyday is a real inconvenience for some people. & 0.582 \\
Do you ever feel hassled about sticking to your treatment plan? & 0.559 \\
\hline
\end{tabular}

(Extraction method: principal components analysis (PCA). One component extracted).

Table 3. The eight-item medication adherence scale.

Items
Do you sometimes forget to take your medications?
People sometimes miss taking their medications for reasons other than forgetting.
Correlation
Thinking over the past two weeks, were there any days when you did not take your medicines?
Have you ever cut back or stopped taking your medication without telling your doctor,
because you felt worse when you took it?
When you travel or leave home, do you sometimes forget to bring along your medications?
Did you take your medications yesterday?
When you feel like your condition is under control, do you sometimes stop taking your medicines?
Taking medication everyday is a real inconvenience for some people.
Do you ever feel hassled about sticking to your treatment plan?
How often do you have difficulty remembering to take all your medications?
0.391




\subsection{MMAS-8 and Other Risk Factors}

It was found that age was related to the overall score $\left(r_{s}=-0.421, p<0.001\right)$, indicating that younger people were more likely to adhere to medication regimen. Gender didn't seem to influence adherence (Mann-Whitney $\mathrm{U}=861.5$, non significant, $\mathrm{r}=-0.19$ ). Kruskal-Wallis tests were also performed for categorical variables, followed by post hoc Mann-Whitney tests using Bonferroni correction. It was found that there were differences between various groups of participants according to marital status $(\mathrm{H}=12.327, p=0.006)$, number of children $(H=9.184, p=0.05)$, level of education $(H=12.092, p=0.007)$, and insurance coverage $(H=11.243, p=0.01)$, but not according to income $(\mathrm{H}=9.221, p=0.101)$. Further, it was noted that married participants had higher adherence scores versus widowers $(\mathrm{U}=373.5, p=0.009, \mathrm{r}=-0.29)$, people who didn't have any children also had higher adherence scores versus participants with 3 or more children $(\mathrm{U}=45.00, p=0.0011, \mathrm{r}=-0.365$ and $\mathrm{U}=10.00, p=0.001, \mathrm{r}=-0.60$, respectively), primary school graduates scored less than higher education graduates $(\mathrm{U}=166.500, p<0.001, \mathrm{r}=0.423)$ and participants who were insured by Agricultural Insurance Organization (OGA) had lower adherence score, compared to people insured by Welfare Organization of Public Servants (OPAD) $(\mathrm{U}=146.00, p=0.009, \mathrm{r}=0.325)$. Representation of relevant data is shown as boxplots in Figures 1-4.

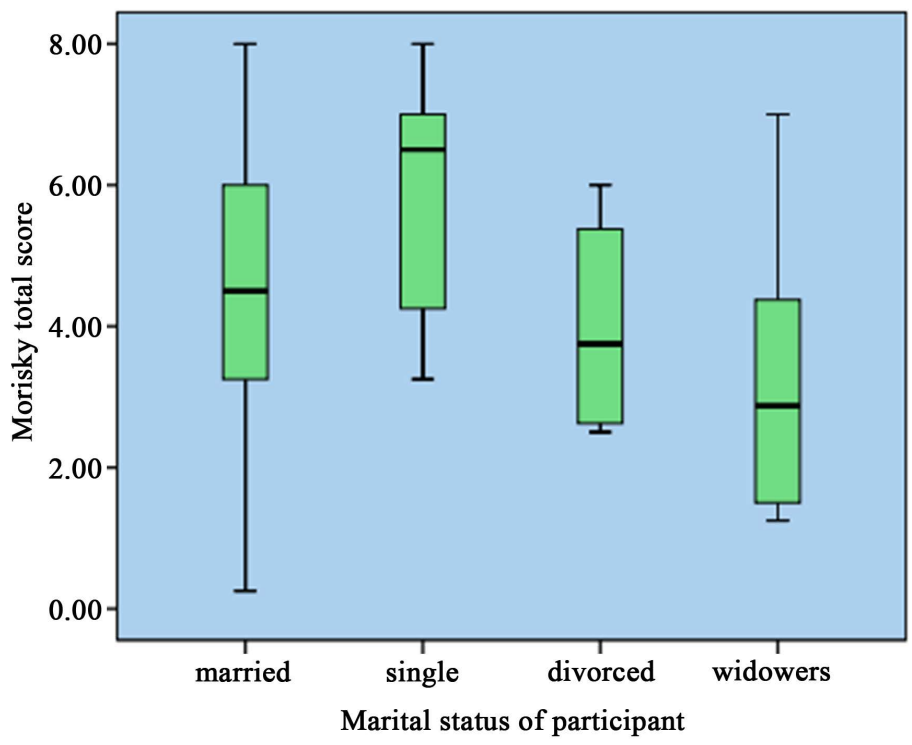

Figure 1. Boxplot of participants' scoring according to their marital status.

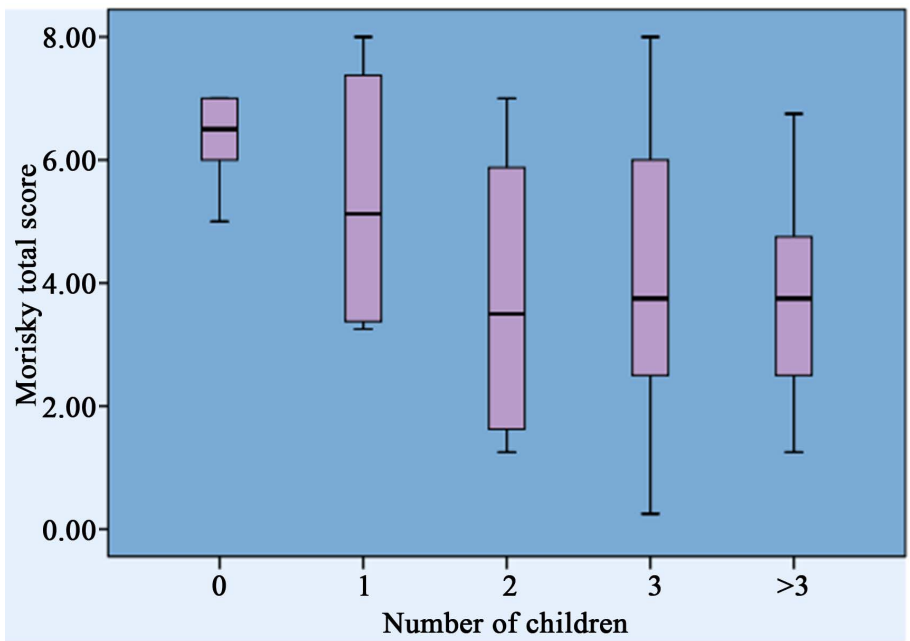

Figure 2. Boxplot of participants' scoring according to number of their children. 


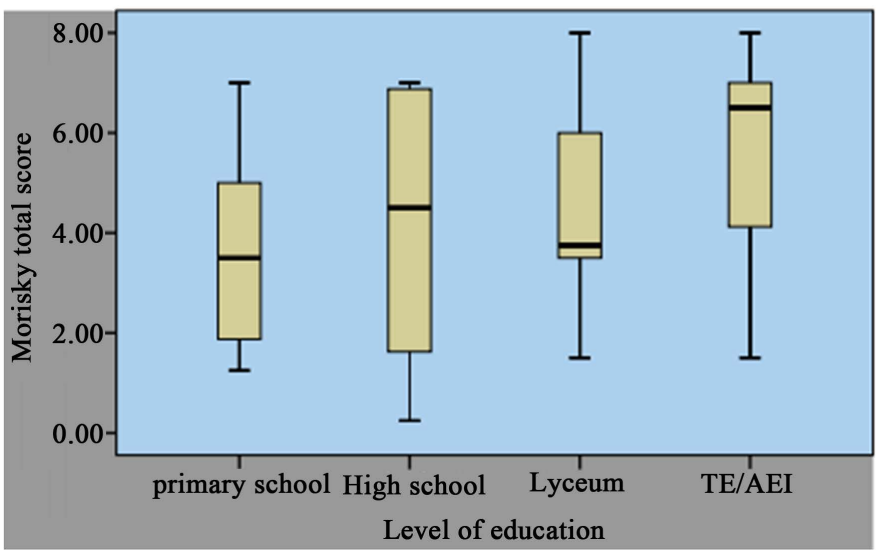

Figure 3. Boxplot of participants’ scoring according to their level of education.

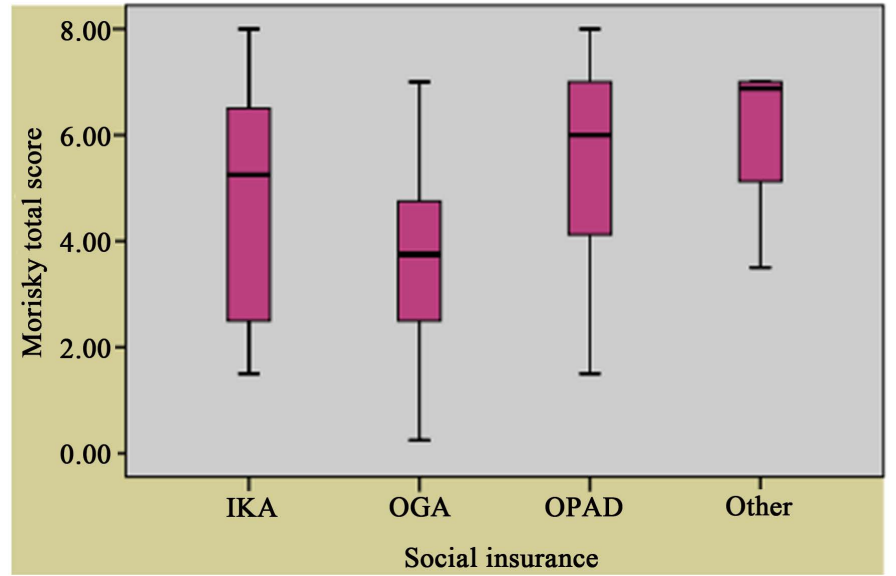

Figure 4. Boxplot of participants' scoring according to their social insurance coverage.

\subsection{Adherence Rates}

According to Morisky et al. (2008), there are three categories of adherent patients: high adherence patients with a score of 8 on the scale, medium adherers with a score of 6 to $<8$, and low adherers with a score of $<6$. According to these cutpoints, the present study population included $72 \%$ low adherers, $25.80 \%$ medium adherers, and $2.2 \%$ high adherers.

\section{Discussion and Conclusion}

\subsection{Discussion}

The aim of this study was to test the psychometric properties of the Greek version of the MMAS-8 in a sample of patients with various chronic illnesses in rural Greece. To the best of our knowledge, the 8-item MMAS has not been used in Greece before. Recently the 8-item MMAS has been validated in many languages across the world. However, all studies validated the scale with patients with a single chronic condition. No previous studies have validated the MMAS-8 in patients with multiple chronic conditions.

The original MMAS-8 study had achieved internal consistency reliability $\alpha=0.83$ [21]. The current study showed satisfactory Cronbach's alpha of 0.753. All items had item to total correlations greater than 0.30 .

Prior published reports on validating the MMAS-8 have reported internal consistency reliability with Cronbach's alpha between 0.54 and 0.79 [20] [25] [28] [29] [32] in hypertensive patients and between 0.61 to 0.675 in patients with type 2 diabetes [14] [24] [27]. Furthermore a validation study with osteoporosis women has reported a Cronbach's alpha of 0.82 [26], while in patients with epilepsy Cronbach's alpha was 0.556 [30] and in 
patients with myocardial infarction Cronbach’s alpha was 0.77 [31].

In relation to construct validity, in our study the scale was unidimensional as all items loaded well on a single factor which was also the case in the original study. This is similar with the studies with hypertensive patients in France and Turkey [20] [32] and on osteoporosis patients in USA [26] and the original validation study of Morisky et al. [21].

However in the studies with type 2 diabetic patients in Thailand and Korea [24] [27] and hypertensive patients in Korea [28] the scale appeared to be 3-dimensional with the dimensions being "forgetting, stopping (intention) and difficulties/barriers”.

Our sample showed intentional non adherence as the study population reported that taking their medication is a hassle for them. A total of $97 \%$ of low adherers feel that it's an inconvenience having to adhere to an intake plan of their pills while $77.6 \%$ choose not to take them and $79.1 \%$ forget to take them. In the same way $95.8 \%$ of medium adherers feel that it's a trouble having to adhere to an intake plan of their pills, $4.2 \%$ choose not to take them and no one forgets to take them. So, in both medium and low adherers (according to Morisky subscales) the most prevalent answer was that they feel that it's a hassle having to adhere to an intake plan of their pills.

In the current study it was not possible to perform test-retest reliability because our sample had a strong willingness to retain anonymity as in rural Greece, research is not common and people tend to mistrust the intentions of researchers. As a result, no contact details were recorded. However several studies conducted in order to validate the 8-item MMAS [27]-[30] have all shown excellent test retest reliability scores.

Convergent validity was supported by a significant correlation between the MMAS-8 total score and the BMQ-Specific Necessity score (Spearman's rho $=0.492, p<0.001$ ). In this study with patients with multiple chronic conditions and on many medications it was expected that the lowest their score on the necessity domain of their beliefs about their medications, the lowest would be their self reported adherence, a finding which is in agreement with the findings of other research [26] [38].

Analysis of risk factors for non-adherence revealed that age was related to adherence with younger patients being more adherent. This is in contrast to other findings elsewhere [14] [20] [24] [39]. However, this might be explained as in our study the older the patients were, the more probable to have multiple chronic conditions and be on a higher number of medications thus reducing adherence as other research in Greece has shown [40]. In addition older age and multiple conditions such as cardiac failure and renal failure usually goes with higher cognitive impairment which is also known to affect adherence [41] [42].

Married people were more adherent than single, which has to do with the social support they receive in a home environment which is in accordance with other findings [9] [43] [39].

Those without children were more adherent than those having 3 or more children probably due to more responsibilities and expenses. Those with low educational level were less adherent than those having a higher educational level, which was also shown in other studies [14] while farmers were less adherent than public servants. This may be linked with their heavy workload and requirements of the farms as well as differences in educational levels.

Low adherence in our study could be attributed to the high percentage of illiterate patients, and the multiple chronic conditions including renal failure and cardiac problems which affect cognitive condition. The low levels of literacy could affect the understanding and comprehension of the survey questions and for that reason the questionnaires were completed with face to face interviews to make sure questions were fully understood.

However the very low percentage of adherent patients in this group is very alarming as other researchers have reported that patients tend to overestimate their degree of adherence on self reported instruments [28].

The current study has several limitations. The findings of this study should be interpreted with caution, since it was conducted with a relatively small sample $(n=100)$, in only one region of Greece and in patients with relatively low income and educational level, possibly rendering the sample not representative of other patients from different socioeconomic or educational backgrounds. Low education level may hinder patient knowledge regarding the likelihood of non adherence to medical regimen. Other limitations are that we did not perform test re-test reliability test for the reasons mentioned above. We considered it more important to get an unbiased indication of adherence rather than risk a social desirability response on MMAS-8. Besides, in every study in every setting, test retest reliability of the MMAS-8 has been found to be excellent. We were also unable to test known group validity with HbA1c levels or with blood pressure control, due to comorbidity of our sample and lack of electronic medical records, which would made it impossible to compare present with previous measure- 
ments.

Another limitation of the study is the lack of comparison between adherence scores and other direct objective ways such as electronic monitoring or pharmacy fill data.

Finally the demographic factors related to adherence should be considered carefully as this was a small scale validation study and further studies are needed. External validity should also be considered with caution as our sample was a self-selected convenience one which limits generalizability.

\subsection{Conclusion}

The current study showed acceptable reliability and validity of the Greek version of the 8-item MMAS to measure adherence to medications for various chronic illnesses. Adherence to medications regimen in Greece appears to be extremely low, thus contributing to increased costs and waste of financial resources. The availability of a brief, easy to administer and valid self reporting adherence scale may provide Greek clinicians the ways to understand non-adherence and furthermore plan appropriate strategies towards appropriate use of medicines thus reducing the costs from the improper use of them. It is strongly recommended that further research on adherence to medications needs to be done in Greece

\section{Funding}

None.

\section{Conflict of Interest}

The authors have no conflict of interest to disclose.

\section{References}

[1] Unni, E.J. and Farris, K.B. (2011) Unintentional Non-Adherence and Belief in Medicines in Older Adults. Patient Education and Counseling, 83, 265-268. http://dx.doi.org/10.1016/j.pec.2010.05.006

[2] Burnier, M. (2006) Medication Adherence and Persistence as the Cornerstone of Effective Antihypertensive Therapy. American Journal of Hypertension, 19, 1190-6. http://dx.doi.org/10.1016/j.amjhyper.2006.04.006

[3] Mardby, A.C., Akerlind, I. and Jorgensen, T. (2007) Beliefs about Medicines and Self-Reported Adherence among Pharmacy Clients. Patient Education and Counseling, 69, 158-164. http://dx.doi.org/10.1016/j.pec.2007.08.011

[4] WHO (2003) Adherence to Long-Term Therapies: Evidence for Action. World Health Organization, Geneva.

[5] Haynes, R.B., Ackloo, E., Sahota, N., McDonald, H.P. and Yao, X. (2008) Interventions for Enhancing Medication Adherence. Cochrane Database of Systematic Reviews, No. 2, Article No.: CD000011. http://dx.doi.org/10.1002/14651858.CD000011.pub3

[6] AlGhurair, S.A., Hughes, C.A., Simpson, S.H. and Guirguis, L.M. (2012) A Systematic Review of Patient Self-Reported Barriers of Adherence to Antihypertensive Medications Using the World Health Organization Multidimensional Adherence Model. The Journal of Clinical Hypertension, 14, 877-886. http://dx.doi.org/10.1111/j.1751-7176.2012.00699.X

[7] Kardas, P., Morrison, V., Fargher, E., Parveen, S., Plumpton, C., Clyne, N., De Geest, S., Dobbels, F., Vrijens, B., Orquhart, J., Lewek, P., Matyjaszczyk, M. and Hughes, D. (2012) Report on the Determinants of Patient Non-Adherence with Short-Term Therapies and Treatment for Chronic Diseases in Europe. In: Ascertaining Barriers for Compliance: Policies for Safe, Effective and Cost Effective Use of Medicines in Europe 2012, 40. http://www.ABCproject.eu

[8] Morrison, V., Fargher, E., Parveen, S., Plumpton, C., Clyne, W., De Geest, S., Dobbels, F., Vrijens, B., Kardas, P. and Hughes, D. (2012) Determinants of Patient Adherence to Antihypertensive Medication: A Multinational Cross Sectional Study. In: Ascertaining Barriers for Compliance: Policies for Safe, Effective and Cost-Effective Use of Medicines in Europe Final Report of the ABC Project 2012.

[9] Maguire, L.K., Hughes, C.M. and McElnay, J.C. (2008) Exploring the Impact of Depressive Symptoms and Medication Beliefs on Medication Adherence in Hypertension-A Primary Care Study. Patient Education and Counseling, 73, 371-376. http://dx.doi.org/10.1016/j.pec.2008.06.016

[10] Stavropoulou, C. (2012) Perceived Information Needs and Non-Adherence: Evidence from Greek Patients with Hypertension. Health Expect, 15, 187-196. http://dx.doi.org/10.1111/j.1369-7625.2011.00679.x

[11] Mastrogiannis, D., Gesouli, E., Mantzorou, M., Noula, M. and Michalitsi, Ch. (2011) Compliance in Hypertensive Pa- 
tients Attending an Athens Hospital. Interscientific Health Care, 3, 14-23.

[12] Yiannakopoulou, E.C., Papadopulos, J.S., Cokkinos, D.V. and Mountokalakis, T.D. (2005) Adherence to Antihypertensive Treatment: A Critical Factor for Blood Pressure Control. European Journal of Cardiovascular Prevention and Rehabilitation, 12, 243-249. http://dx.doi.org/10.1097/00149831-200506000-00010

[13] Hodgson, T. and Cai, L. (2001) Medical Care Expenditure for Hypertension, Its Complications and Its Comorbidities. Medical Care, 39, 599-615. http://dx.doi.org/10.1097/00005650-200106000-00008

[14] Al-Qazaz, H.K., Hassali, M.A., Shafie, A.A., Sulaiman, S.A., Sundram, S. and Morisky, D.E. (2010) The Eight-Item Morisky Medication Adherence Scale MMAS: Translation and Validation of the Malaysian Version. Diabetes Research and Clinical Practice, 90, 216-221. http://dx.doi.org/10.1016/j.diabres.2010.08.012

[15] Vandoros, S. and Stargardt, T. (2013) Reforms in the Greek Pharmaceutical Market during the Financial Crisis. Health Policy, 109, 1-6. http://dx.doi.org/10.1016/j.healthpol.2012.08.016

[16] Karamanoli, E. (2012) Greece’s Financial Crisis Dries up Drug Supply. The Lancet, 379, 302. http://dx.doi.org/10.1016/S0140-6736(12)60129-9

[17] Koschack, J., Marx, G., Schnakenberg, J., Kochen, M.M. and Himmel, W. (2010) Comparison of Two Self-Rating Instruments for Medication Adherence Assessment in Hypertension Revealed Insufficient Psychometric Properties. Journal of Clinical Epidemiology, 63, 299-306. http://dx.doi.org/10.1016/j.jclinepi.2009.06.011

[18] Wang, Y., Kong, M.C. and Ko, Y. (2012) Psychometric Properties of the 8-Item Morisky Medication Adherence Scale in Patients Taking Warfarin. Thrombosis and Haemostasis, 108, 789-795. http://dx.doi.org/10.1160/TH12-05-0368

[19] Fargher, E. and Hughes, D. (2012) Report on the Cost Effectiveness of Interventions That Promote Adherence: A Systematic Review of the Literature and Economic Evaluation. In Ascertaining Barriers for Compliance: Policies for Safe, Effective and Cost-Effective Use of Medicines in Europe Final Report of the ABC Project. Accessed 20th December 2012. http://www.ABCproject.eu

[20] Korb-Savoldell, V., Gillaizeau, F., Pouchot, J., Lenain, E., Postel-Vinay, N., Plouin, P.F., Durieux, P. and Sabatier, B. (2012) Validation of a French Version of the 8-Item Morisky Medication Adherence Scale in Hypertensive Adults. The Journal of Clinical Hypertension, 14, 429-434. http://dx.doi.org/10.1111/j.1751-7176.2012.00634.x

[21] Morisky, D.E., Ang, A., Krousel-Wood, M. and Ward, H. (2008) Predictive Validity of a Medication Adherence Measure for Hypertension Control. The Journal of Clinical Hypertension, 10, 348-354. http://dx.doi.org/10.1111/j.1751-7176.2008.07572.x

[22] Krousel-Wood, M., Islam, T., Webber, L.S., Re, R.N., Morisky, D.E. and Muntner, P. (2009) New Medication Adherence Scale versus Pharmacy Fill Rates in Seniors with Hypertension. American Journal of Managed Care, 15, 59-66.

[23] Morisky, D.E. and DiMatteo, M.R. (2011) Improving the Measurement of Self-Reported Medication Nonadherence: Response to Authors. Journal of Clinical Epidemiology, 64, 262-263. http://dx.doi.org/10.1016/j.jclinepi.2010.09.010

[24] Sakthong, P., Chabunthom, R. and Charoenvisuthiwongs, R. (2009) Psychometric Properties of the Thai Version of the 8-Item Morisky Medication Adherence Scale in Patients with Type 2 Diabetes. Annals of Pharmacotherapy, 43, 950957. http://dx.doi.org/10.1345/aph.1L453

[25] Saleem, F., Hassali, M.A., Akmal, S., Morisky, D.E., Atif, M., Al-Qazaz, H.K., et al. (2012) Translation and Validation Study of Morisky Medication Adherence Scale (MMAS): The Urdu Version for Facilitating Person-Centered Healthcare in Pakistan. The International Journal of Person Centered Medicine, 2, 384-390.

[26] Reynolds, K., Viswanathan, H.N., O’Malley, C.D., Muntner, P., Harrisn, T.N., Cheetham, T.C., Hsu, J.W., Gold, D.T., Silverman, S., Grauer, A. and Morisky, D.E. (2012) Psychometric Properties of the Osteoporosis-Specific Morisky Medication Adherence Scale in Postmenopausal Women with Osteoporosis Newly Treated with Bisphosphonates. Annals of Pharmacotherapy, 46, 659-670. http://dx.doi.org/10.1345/aph.1Q652

[27] Lee, W.Y., Ahn, J., Kim, J.H., Hong, Y.P., Hong, S.K., Kim, Y.T., Lee, S.H. and Morisky, D.E. (2013) Reliability and Validity of a Self-Reported Measure of Medication Adherence in Patients with Type 2 Diabetes Mellitus in Korea. Journal of International Medical Research, 41, 1098-1110. http://dx.doi.org/10.1177/0300060513484433

[28] Kim, J.H., Lee, W.Y., Hong, Y.P., Ryu, W.S., Lee, K.J., Lee, W.S. and Morisky, D.E. (2014) Psychometric Properties of a Short Self-Reported Measure of Medication Adherence among Patients with Hypertension Treated in a Busy Clinical Setting in Korea. Journal of Epidemiology, 24, 132-140. http://dx.doi.org/10.2188/jea.JE20130064

[29] de Oliveira-Filho, A.D., Morisky, D.E., Neves, S.J.F., Costa, F.A. and de Lyra Jr., D.P. (2014) The 8-Item Morisky Medication Adherence Scale: Validation of a Brazilian-Portuguese Version in Hypertensive Adults. Research in Social and Administrative Pharmacy, 10, 554-561. http://dx.doi.org/10.1016/j.sapharm.2013.10.006

[30] Yang, A., Wang, B., Zhu, G., Jiao, Z., Fang, Y., Tang, F., Ma, C., Zhao, Y., Cheng, C. and Zhong, M. (2014) Validation of Chinese Version of the Morisky Medication Adherence Scale in Patients with Epilepsy. Seizure, 23, $295-299$. http://dx.doi.org/10.1016/j.seizure.2014.01.003 
[31] Yan, J., You, L.M., Yang, Q., Liu, B., Jin, S., Zhou, J., Lin, C. and Morisky, D.E. (2014) Translation and Validation of a Chinese Version of the 8-Item Morisky Medication Adherence Scale in Myocardial Infarction Patients. Journal of Evaluation in Clinical Practice, 20, 311-317. http://dx.doi.org/10.1111/jep.12125

[32] Asilar, R.H., Gozum, S., Capic, C. and Morisky, D.E. (2014) Reliability and Validity of the Turkish Form of the Eight-Item Morisky Medication Adherence Scale in Hypertensive Patients. Anadolu Kardiyoloji Dergisi, 14, $692-700$.

[33] Hutcheson, G. and Sofroniou, N. (1999) The Multivariate Social Scientist. Sage, London.

[34] Horne, R., Weinman, J. and Hankins, M. (1999) The Beliefs about Medicines Questionnaire: The Development and Evaluation of a New Method for Assessing the Cognitive Representation of Medication. Psychology \& Health, 14, 1-24. http://dx.doi.org/10.1080/08870449908407311

[35] Horne, R. and Weinman, J. (2002) Self-Regulation and Self-Management in Asthma: Exploring the Role of Illness Perceptions and Treatments Beliefs in Explaining Non-Adherence to Preventer Medication. Psychology \& Health, 17, 17-32. http://dx.doi.org/10.1080/08870440290001502

[36] Horne, R. and Weinman, J. (1999) Patients' Beliefs about Prescribed Medicines and Their Role in Adherence to Treatment in Chronic Physical Illness. Journal of Psychosomatic Research, 47, 555-567. http://dx.doi.org/10.1016/S0022-3999(99)00057-4

[37] Aletras, V., Zacharaki, F. and Niakas, D. (2007) Questionnaire for the Measurement of Outpatient Satisfaction in the Ophthalmology Clinic of a Greek Public Hospital. Archives of Hellenic Medicine, 24, 89-96.

[38] Schüz, B., Marx, C., Wurm, S., Warner, L.M., Ziegelmann, J.P., Schwarzer, R. and Tesch-Römer, C. (2011) Medication Beliefs Predict Medication Adherence in Older Adults with Multiple Illnesses. Journal of Psychosomatic Research, 70, 179-187. http://dx.doi.org/10.1016/j.jpsychores.2010.07.014

[39] Stavropoulou, C. (2011) Non-Adherence to Medication and Doctor-Patient Relationship: Evidence from a European Survey. Patient Education and Counseling, 83, 7-13. http://dx.doi.org/10.1016/j.pec.2010.04.039

[40] Tsiantou, V., Pantzou, P., Pavi, E., Koulierakis, G. and Kyriopoulos, J. (2010) Factors Affecting Adherence to Antihypertensive Medication in Greece: Results from a Qualitative Study. Patient Preference and Adherence, 4, 335-343. http://dx.doi.org/10.2147/PPA.S12326

[41] Theofilou, P. (2012) Factors Affecting Level of Compliance in Chronic Patients. Internal Medicine, 2, e106. http://dx.doi.org/10.4172/2165-8048.1000e106

[42] Lin, Y.S., Ho, Y., Hu, C.J., Su, W.W., Hsu, K.Y., Shen, W.W., Chiueh, C.C. and Yuan, R.Y. (2013) Development of a Taiwan Version of the Eight-Item Morisky Medication Adherence Scale and Factors Influencing Patients' Comprehension. Journal of Experimental \& Clinical Medicine, 5, 77-80. http://dx.doi.org/10.1016/j.jecm.2013.02.005

[43] Sweileh, W.M., Sa’ed, H.Z., Nab’a, R.J.A., Deleq, M.I., Enaia, M.I., Sana’a, M.N. and Al-Jabi, S.W. (2014) Influence of Patients' Disease Knowledge and Beliefs about Medicines on Medication Adherence: Findings from a Cross-Sectional Survey among Patients with Type 2 Diabetes Mellitus in Palestine. BMC Public Health, 14, 94.

http://dx.doi.org/10.1186/1471-2458-14-94 